INPLASY

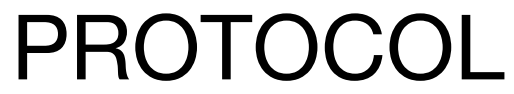

To cite: Ren et al. Clinical efficacy comparison of metformin and Myo-inositol in the treatment of polycystic ovary syndrome: a systematic review and meta-analysis protocol. Inplasy protocol 202220013. doi:

10.37766/inplasy2022.2.0013

Received: 06 February 2022

Published: 06 February 2022

Corresponding author: Jian Ren

1961810929@qq.com

Author Affiliation:

Shandong University of Traditional Chinese Medicine.

Support: The Project of National Natura.

Review Stage at time of this submission: Data analysis.

Conflicts of interest:

None declared.

\section{Clinical efficacy comparison of metformin and Myo-inositol in the treatment of polycystic ovary syndrome: a systematic review and meta-analysis protocol}

\author{
Ren, J1; Xu, Y2; Liang, C3; Sun, Y4.
}

Review question / Objective: Which drug is better for treating polycystic ovary syndrome than metformin or Myo-inositol? Condition being studied: Polycystic ovary syndrome; metformin; myo-inositol.

Information sources: We will search PubMed, the Cochrane Library, The Web of Science, and EMBASE for qualified RCTS in these databases. We will also introduce the search strategy of PubMed in detail.

INPLASY registration number: This protocol was registered with the International Platform of Registered Systematic Review and Meta-Analysis Protocols (INPLASY) on 06 February 2022 and was last updated on 06 February 2022 (registration number INPLASY202220013).

\section{INTRODUCTION}

Review question / Objective: Which drug is better for treating polycystic ovary syndrome than metformin or Myo-inositol?

Condition being studied: Polycystic ovary syndrome; metformin; myo-inositol.

\section{METHODS}

Participant or population: PCOS patients were diagnosed according to the diagnostic criteria of PCOS formulated by ESHRE/ASRM in Rotterdam in 2003.

Intervention: The experimental group was treated with metformin. 
Comparator: The control group was treated with MyO-inositol.

Study designs to be included: This study included randomized controlled trials (RCTS) of two drugs for PCOS. We will exclude all non-randomized controlled trials, such as review, non-clinical trials.

Eligibility criteria: This study included randomized controlled trials (RCTS) of two drugs for PCOS. We will exclude all nonrandomized controlled trials, such as review, non-clinical trials and uncontrolled trials.

Information sources: We will search PubMed, the Cochrane Library, The Web of Science, and EMBASE for qualified RCTS in these databases. We will also introduce the search strategy of PubMed in detail.

Main outcome(s): Results: FSH, LH, LH/ FSH, insulin, HOMA, insulin, ovarian volume, testosterone, progesterone, prolactin, dehydroepiandrosterone, FBS, 17-hydroxyprogesterone (17-OH-P), androgen, estradiol andSHBG.

Quality assessment / Risk of bias analysis: Two researchers independently performed data extraction and quality assessment based on screening criteria, and crosschecked. If there is any conflict of opinion, it will be solved through collective discussion of the research group. In the process of literature screening, irrelevant titles were eliminated, abstracts and full texts were further read to determine the final inclusion of literature. Basic information (first author, year of publication, number of patients, interventions, and course of treatment) was extracted from the included studies. These include menstrual timing, age, BMI, FSH, $\mathrm{LH}$, insulin, FBS, LH/FSH, testosterone, HOM A, ovarian capacity, dehydroepiandrosterone, progesterone, 17OH-P, prolactin, SHBG, estradiol and androgen.

Strategy of data synthesis: Meta-analyses were performed using RevMan 5.3 and Stata 12.0, respectively. The odds ratio was calculated for dichotomous variables and the mean and standard deviation were calculated for continuous variables. Both groups (metformin and inositol) were presented with $95 \%$ confidence intervals, and data heterogeneity was assessed using Q-test and II. Sensitivity analysis is required to determine the reliability of the results. All results were presented in the form of forest map, and Begg and Egger tests were used as statistical tests for publication bias of test results.

Subgroup analysis: Subgroup analysis was performed if there was significant heterogeneity between the included studies and if it would be significantly reduced by subgrouping at different ages or BMI, or by appropriate interventions.

Sensitivity analysis: A sensitivity analysis will be conducted to test the robustness of study findings by eliminating low quality studies.

Country(ies) involved: China.

Keywords: Polycystic ovary syndrome; Metformin; Myo - inositol. Clinical efficacy.

Contributions of each author:

Author 1 - Jian Ren.

Author 2 - Yan Xu.

Author 3 - Chengfeng Liang.

Author 4 - Yu Sun. 